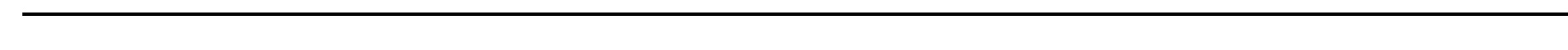

$-2$ 


\section{Highly oxygenated molecules from atmospheric autoxidation of hydrocarbons: A prominent challenge for chemical kinetics studies}

Mikael Ehn ${ }^{1}$, Torsten Berndt ${ }^{2}$, Jürgen Wildt ${ }^{3}$, Thomas M entel ${ }^{4}$

${ }^{1}$ Department of Physics, P.O. Box 64, 00014 University of Helsinki, Finland.

${ }^{2}$ Institute for Tropospheric Research (TROPOS), 04318 Leipzig, Germany.

${ }^{3}$ Institute of Bio- and Geosciences (IBG-2), Forschungszentrum Jülich, 52425 Jülich, Germany.

${ }^{4}$ Institute for Energy and Climate Research (IEK-8), Forschungszentrum Jülich, 52425 Jülich, Germany.

Author email: mikael.ehn@helsinki.fi

\section{Abstract}

Recent advances in chemical ionization mass spectrometry have allowed the detection of a new group of compounds termed highly oxygenated molecules (HOM). These are atmospheric oxidation products of volatile organic compounds (VOC) retaining most of their carbon backbone, and with O/C-ratios around unity. Due to their surprisingly high yields and low vapor pressures, the importance of HOM for aerosol formation has been easy to verify. However, the opposite can be said concerning the exact formation pathways of HOM from major aerosol precursor VOC. While the role of peroxy radical autoxidation, i.e. consecutive intra-molecular $\mathrm{H}$-shifts followed by $\mathrm{O}_{2}$ addition, has been recognized, the detailed formation mechanisms remain highly uncertain. A primary reason is that the autoxidation process occurs on subsecond timescales and is extremely sensitive to environmental conditions like gas composition, temperature and pressure. This, in turn, poses a great challenge for chemical kinetics studies to be able to mimic the relevant atmospheric reaction pathways, while simultaneously using conditions suitable for studying the short-lived radical intermediates. In this perspective, we define six specific challenges for this community to directly observe the initial steps of atmospherically relevant autoxidation reactions and thereby facilitate vital improvements in the understanding of VOC degradation and organic aerosol formation.

\section{Introduction}

Both biogenic and anthropogenic sources constantly emit volatile organic compounds (VOC) into the atmosphere [1,2]. These emissions strongly impact atmospheric chemistry, human health and Earth's climate, both by themselves and through their potential reaction products. The major reaction pathway for VOC in the atmosphere is oxidation to form oxidized VOC (OVOC). Excluding subsequent fragmentation reactions, increased oxidation levels decrease the volatility of the OVOC. If reaching low enough vapor 
pressures, the OVOC can condense onto airborne particulate matter, contributing to secondary organic aerosol (SOA) in the atmosphere.

While the ubiquity and global importance of SOA has been shown $[3,4]$, open questions remain concerning the formation and evolution of atmospheric SOA. One long-standing issue is that laboratory studies tend to under-predict both the amount and the level of oxidation of SOA commonly observed in the atmosphere $[3,5]$. Until recently, the only possible mechanism by which the observed high $\mathrm{O} / \mathrm{C}$-ratios, and coincident low volatility, could be explained was thought to be consecutive oxidation reactions, primarily by the hydroxyl radical, OH [6,7]. Similarly, the majority of SOA was long thought to be produced predominantly by semi-volatile organic compounds (SVOC), though studies were emerging which showed that the volatility of SOA needed to be much lower [8,9]. However, no formation mechanisms were known to produce such (extremely) low-volatile organic compounds, (E)LVOC [10].

The first observational indications of an until then unknown class of low-volatile compounds came from the boreal forest [11] and later under controlled conditions in the laboratory [12], where highly oxygenated molecules (HOM ) were detected from biogenic VOC, monoterpenes $\left(\mathrm{C}_{10} \mathrm{H}_{16}\right)$, upon oxidation by ozone. The formation mechanism of HOM remained elusive until nitrate-ion based chemical ionization mass spectrometry [13] improved the detection and quantification of HOM and allowed probing the formation pathways under well-controlled conditions [14]. The key process was found to be peroxy radical $\left(\mathrm{RO}_{2}\right)$ autoxidation $[15,16]$, i.e. intramolecular $\mathrm{H}$-shifts followed by rapid addition of $\mathrm{O}_{2}$ forming a more oxidized peroxy radical. This process was known to take place in the condensed phase as well as at elevated temperatures like those found in combustion processes (where it leads to so-called degenerate chain branching causing autoignition) $[17,18]$. Single steps had also been suggested to take place under atmospheric conditions $[15,18]$, but the possibility of multiple subsequent autoxidation steps had not been addressed, as many of the studied systems were small molecules where this process was unlikely proceed.

In recent years, the importance of HOM in the atmosphere have received much interest, with studies finding that they can have significant impacts on both aerosol particle number $[19,20]$ and mass $[14,21]$. Detailed studies have also been performed to elucidate the formation pathways of HOM [22,23], but current techniques for HOM production and detection have several limitations. These limitations are currently the primary restriction for attempts to further our understanding of SOA formation and consequent health and climate impacts.

The aim of this perspective is to highlight the main challenges for achieving a mechanistic understanding of atmospheric HOM formation, setting the challenge to the chemical kinetics community to quantify the key processes involved. In this context, we define the term "HOM " as highly oxidized gas phase products of peroxy radical autoxidation from VOC, formed without considerable loss of carbon.

\section{Peroxy radical autoxidation in the atmosphere}

The average lifetime of $\mathrm{RO}_{2}$ with respect to bimolecular pathways in the atmosphere ranges from seconds to minutes, depending on the $\mathrm{RO}_{2}$ reactivity and the concentration of reaction partners, primarily $\mathrm{NO}, \mathrm{HO}_{2}$ and other $\mathrm{RO}_{2}$. For autoxidation to have any practical relevance in the atmosphere, the intramolecular $\mathrm{H}$ shift reactions by $\mathrm{RO}_{2}$ need to take place on these same (or shorter) timescales. Autoxidation can also proceed via endo-cyclization in unsaturated $\mathrm{RO}_{2}$ radicals, as it allows subsequent $\mathrm{O}_{2}$-addition, similar to 
H-shifts. For pure hydrocarbons without functional groups, H-abstractions are mostly slower (e.g. Glowacki \& Pilling [18] and references therein) and since these were typically used as test compounds in earlier studies [24], autoxidation was not thought to take place under atmospheric conditions. However, functionalization can greatly weaken $\mathrm{C}-\mathrm{H}$ bonds, and certain molecules are prone to form radicals where $\mathrm{H}$-shift reactions can take place on sub-second time scales. One such example is the ozonolysis of endocyclic alkenes under atmospheric conditions, where the initial $\mathrm{RO}_{2}$ formed after ring-opening can often internally abstracts an $\mathrm{H}$-atom from an aldehydic carbon $[23,25]$. For such molecules, $\mathrm{H}$-shifts and subsequent fast addition of $\mathrm{O}_{2}$, i.e. autoxidation, can easily compete with bimolecular termination pathways for $\mathrm{RO}_{2}$. Comparison to ozonolysis of linear alkenes with different substitutions at the tail clearly pointed out the importance of substitution, specifically by aldehyde groups, for $\mathrm{H}$-shifts and the entire autoxidation process [23].

The seemingly late recognition of the importance of autoxidation in atmospheric VOC oxidation has several reasons. Although the choice of unrepresentative model compounds in earlier studies contributed, the primary reason was the lack of suitable instruments for the direct detection of the HOM products. In the next two subsections, we first describe the state-of-the-art methods with which the important findings were made possible, followed by discussion on specific systems in which autoxidation has been observed and what is currently known about the process.

\section{Observational methods and limitations}

The HOM products resulting from consecutive autoxidation steps will typically contain several hydroperoxide moieties as well as carbonyl and/or alcohol groups. Such molecules are expected to be both reactive and low-volatile. These features make them important for SOA formation, but at the same time makes their detection extremely challenging as they are efficiently removed from the gas phase upon contact with any surface. The observational breakthrough came from recent instrumental developments in the field of mass spectrometry, designed to measure other short-lived and easily lost atmospheric trace species, namely ambient ions [26] and sulfuric acid [13]. The ions were measured without any pretreatment using an atmospheric pressure interface time-of-flight (APi-TOF) mass spectrometer, while the sulfuric acid measurements relied on the same instrument with an added chemical ionization $(\mathrm{Cl})$ source (Cl-APi-TOF). The $\mathrm{Cl}$ source has been used with $\mathrm{NO}_{3}{ }^{-}$as reagent ion in most studies $[14,19,20,25,27-30]$ due to its selectivity towards $\mathrm{HOM}$. The detected ions are primarily clusters of $\mathrm{HOM}$ with $\mathrm{NO}_{3}{ }^{-}$, formed during the $\sim 200$ ms reaction time between sample and reagent ions. M ore details on the working principle of the Cl-APi-TOF can be found elsewhere $[13,14]$.

The first HOM observation was made from naturally charged ions using the APi-TOF [11,12], but the quantification enabled by the active charging in the Cl-APi-TOF was the primary driver for the advances over the last years. The $\mathrm{NO}_{3}{ }^{-} \mathrm{Cl}$-APi-TOF also provided one more critical piece of information which helped to clarify the role of autoxidation, namely the direct observation of highly oxygenated precursor $\mathrm{RO}_{2}$. For example, in a systematic study using ozonolysis of cycloalkenes $\left(C_{5}-C_{7}\right)$ and methyl-substituted cyclohexenes, patterns were observed of $\mathrm{HOM}-\mathrm{RO}_{2}$ separated by 32 mass units $\left(\mathrm{O}_{2}\right)$, as expected for the autoxidation process [23]. Similarly, their respective termination products were also observed: carbonyl compounds ( -17 mass units), alcohols ( -15 mass unit) and hydroperoxides ( +1 mass unit). affinit

The detection of $\mathrm{HOM}$ by clustering with $\mathrm{NO}_{3}{ }^{-}$typically requires the formation of two hydrogen bonds between the HOM and the reagent ion [31], which in practice means that this method primarily detects molecules with 6 or more 0 -atoms. In order to detect also less oxygenated compounds, the CI-APi-TOF 
has in some cases been deployed using acetate as the reagent ion $[32,33]$. This has allowed the detection of less oxidized products all the way down to the initial $\mathrm{RO}_{2}$ radicals. The drawback of this method is the decreased selectivity, which makes it far more susceptible to other more abundant species masking out the HOM signals. Therefore, current use has been limited to ultra-clean laboratory conditions.

Other experimental techniques have also been able to present signals of HOM that may be related to autoxidation products both in the gas-phase and in aerosols [34-38]. Consolidating these measurements with those of the $\mathrm{Cl}-\mathrm{APi}$-TOF remain an important topic.

Whatever technique one applies to HOM detection, one critical limitation remains: the lack of authentic standards. The HOM are expected to be highly reactive poly-peroxides, and as such cannot be easily synthesized and handled as reference substances. This substantially limits the quantification of these molecules, and relatively large uncertainties are always inherent. However, the sensitivity towards HOM can be estimated based on other compounds, and kinetic limitations such as maximum collision rates can be used to infer upper and lower bounds for the observed concentrations. An equally important constraint arises from the use of mass spectrometry, where elemental compositions can be identified, but molecular structure remain elusive. This severely hampers the mechanistic understanding of HOM formation. Structural information can be inferred from well-designed experiments where changes are monitored as a function of added reactants, such as NO [14,22]. Other useful methods to obtain structural information are the addition of heavy water $\left(D_{2} \mathrm{O}\right)$ to study the number of labile $\mathrm{H}$-atoms by $\mathrm{H} \neq \mathrm{D}$ exchange, and the use of isotopic labeling [25].

If structural information of reaction intermediates were obtainable, it would still require fast enough measurements. Currently, sub-second processes are not measurable using available methods for HOM detection. This is in contrast to studies of low-temperature combustion processes where $\mathrm{H}$-shifts and $\mathrm{O}_{2}$ additions have been successfully studied on millisecond timescales using pulsed laser initiation and direct photoionization by synchrotron-generated vacuum ultraviolet radiation [39]. Whether such methods can be used for studies relevant for atmospheric processes remains to be seen. To our knowledge, such efforts have so far not been undertaken, but they may eventually provide an essential way forward by facilitating both faster measurements and more selective observations of radical intermediates.

New experimental capabilities opened up this new research field of atmospheric autoxidation and HOM formation, but they were accompanied by computational support for the evaluation of reaction mechanisms. For example, quantum chemical (QC) calculations have facilitated progress in $\mathrm{H}$-shift rate estimates [25] as well as our understanding of both Cl-APi-TOF charging probabilities [31] and HOM volatility estimates [40].

\section{Atmospheric VOCs and HOM formation}

HOM formation by autoxidation has been observed from a variety of atmospherically emitted molecules under various conditions. Most investigations have involved monoterpenes, the second most emitted VOC globally [1], and important contributors to biogenic SOA. But also other biogenic and anthropogenic emissions have been studied and found to produce HOM. Below, we present the most important VOC and describe the current knowledge of their HOM formation mechanisms, starting with simpler surrogate molecules. 


\section{Surrogate molecules: cyclic alkenes}

Three of the most abundant monoterpenes (. -pinene, limonene, carene) all contain a six-membered ring with an endo-cyclic double bond. Therefore, cyclohexene and methyl cyclohexenes have been used as surrogate molecules for the more complex monoterpenes in many types of studies. In HOM formation studies specifically, $\mathrm{C}_{5}-\mathrm{C}_{8}$ cyclic alkenes have all been utilized and found to yield radical and closed shell products through similar pathways [23,33]. Figure 1 depicts an example pathway for HOM formation following ozonolysis of cyclic alkenes. The autoxidation can be terminated at each peroxy radical (S1-S4), but Fig.1 only shows example termination products (P1-P5) for the most highly oxidized $\mathrm{RO}_{2}$ (S4).

Cyclohexene is a symmetric molecule which provides a small enough amount of possible reaction pathways that QC calculations can try to estimate the process step-by-step. Reasonable agreement was found with experiments conducted in a flow tube [25], with calculations finding fast enough autoxidation pathways that they could take place on the time scales of the experiments (seconds to tens of seconds. Kinetic experiments on the reaction of $\mathrm{HOM}-\mathrm{RO}_{2}$ radicals with $\mathrm{NO}, \mathrm{NO}_{2}$ and $\mathrm{SO}_{2}$ revealed a similar reactivity toward these trace gases as known from "normal" $\mathrm{RO}_{2}$ in the literature [33].

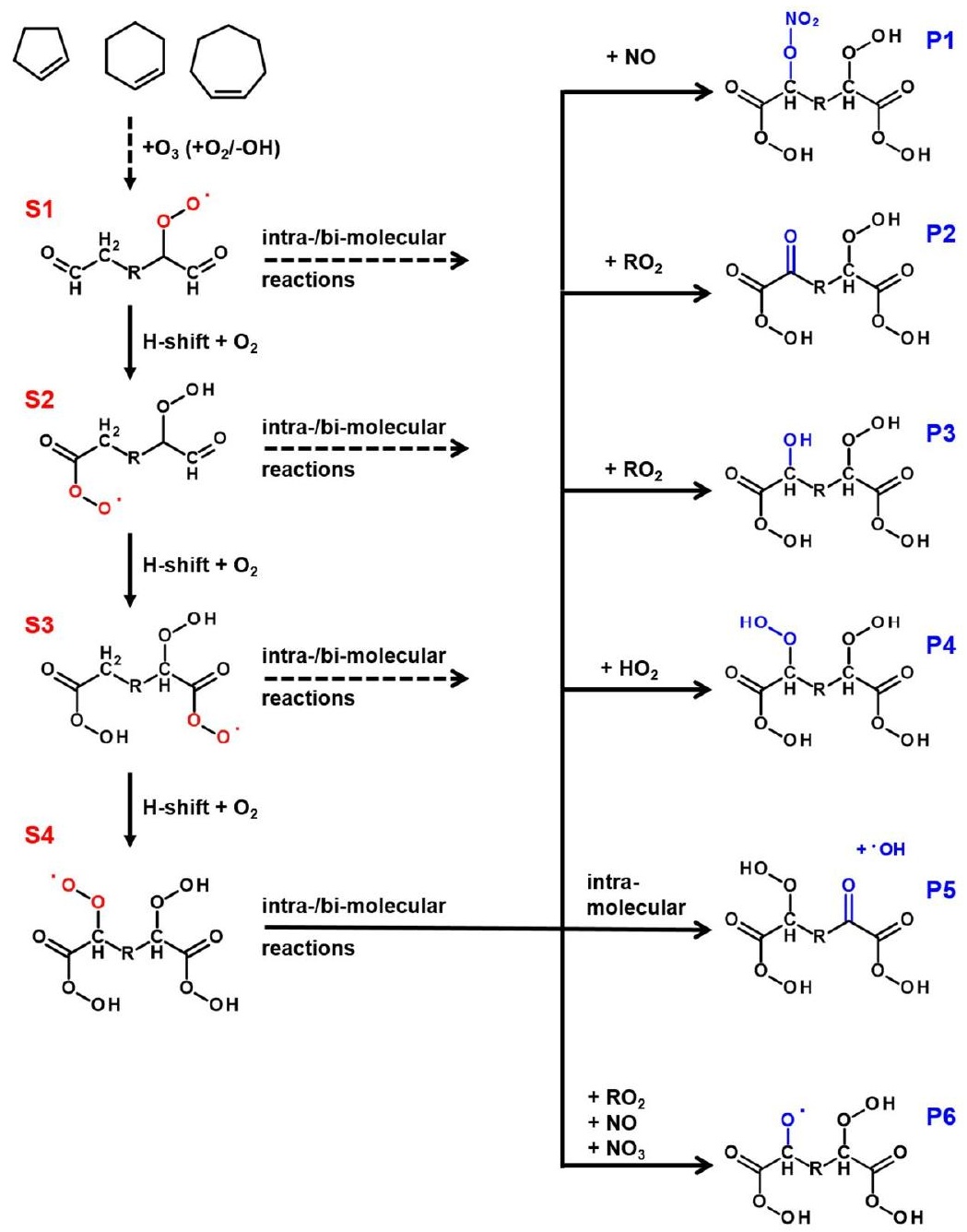

Figure 1. General schematic of autoxidation and HOM formation following ozonolysis of cyclic alkenes. The Criegee and vinyl hydroperoxide intermediates which follow the initial ozone reaction are not 
shown, and the scheme starts from the first peroxy radical (S1). This radical can undergo autoxidation to form more oxidized $\mathrm{RO}_{2}$ (S2-S4). Autoxidation competes with uni- and bimolecular reactions for each $\mathrm{RO}_{2}$. Here typical HOM product types (P1-P5) are only shown from S4. Radical propagation can also proceed via alkoxy radical pathways (P6).

\section{M onoterpenes}

The largest HOM yields reported to date have been measured during ozonolysis of the monoterpenes $r$ pinene and limonene. Molar HOM yields for $r$-pinene have ranged from 3-7 \% while for limonene the reported range has been 5-17\%. These values are based on both flow tube studies, where the reaction time is on the order of seconds [21], as well as chamber studies with reaction times on the order of an hour [14]. In other words, the variability includes both the measurement uncertainty as well as the possibility of further reactions in the chambers. The latter being especially likely in the case of limonene which contains an additional exo-cyclic double bond.

HOM yields from the ozonolysis of monoterpenes without endo-cyclic double bonds have been reported for myrcene $(0.5 \%$ ) and $t$-pinene (G0.1\%) [21]. The reaction with ozone will in these cases lead to cleavage of the molecule and the resulting $\mathrm{RO}_{2}$ may not have any easily accessible $\mathrm{H}$-atoms to abstract. This limits the potential of autoxidation reactions which in turn helps explain the lower yields compared to $r$-pinene and limonene.

While the step-by-step progression of the autoxidation may be understood for cyclohexene, even for the most studied monoterpene, $r$-pinene, the specific pathways to HOM remain elusive. Even when considering all different $\mathrm{RO}_{2}$ expected to form in the initial ozonolysis reaction, $\mathrm{QC}$ calculations suggest that the still-intact four-membered ring produces a steric hindrance for $\mathrm{H}$-abstractions [41]. As high HOM yields have been observed from $r$-pinene, this suggests unaccounted for reaction pathways available for the molecule. This is further supported by a surprisingly low number of labile H-atoms observed in $r$ pinene ozonolysis products [27].

While the above related to ozonolysis, also hydroxyl radical oxidation studies have been performed with monoterpenes. In the most comprehensive study [21], flow tube experiments found HOM yields of 0.5$1.0 \%$ for four different monoterpenes, with decreased yields for the ones with endo-cyclic double bonds and increased for the other two, when comparing to ozonolysis reactions. However, a later study using acetate CIMS [32] has shown that the previously reported OH-derived HOM yields have likely been underestimated. Compared to the ozonolysis case, the structurally different $\mathrm{OH}$-derived HOM seem to form generally less stable clusters with $\mathrm{NO}_{3}{ }^{-}$, decreasing the sensitivity as the assumption of collisionlimited charging no longer holds. While some proposed structures for HOM from the OH-initated - - and -pinene oxidation have been proposed, the bicyclic structure of these monoterpenes causes inevitable steric effects that can hamper the autoxidation and the exact pathways remain ambiguous.

\section{Other precursors: Sesquiterpenes, isoprene and aromatics}

For sesquiterpenes, only a limited number of HOM formation studies are available in the literature $[42,43]$. Reported molar HOM yields from ozonolysis span a range of 0.6-1.8 \%. The detected HOM product distribution of sesquiterpenes with multiple double bonds, for instance b-caryophyllene, point to more complex reaction schemes, most likely including endo-cyclization steps [44]. A recent study on unimolecular pathways of unsaturated $\mathrm{RO}_{2}$ radicals shows that the rate of endo-cyclization steps can be faster than the internal $\mathrm{H}$-atom abstraction [45]. 
In the case of isoprene, despite the huge emission strength and its general importance for atmospheric chemistry, very little is known about possible $\mathrm{HOM}$ formation. For $\mathrm{RO}_{2}$ from $\mathrm{OH}$ radical-initiated reactions, theoretical calculations predict efficient 1,6 $\mathrm{H}$-shifts that lead to products bearing up to 70 -atoms [46]. Reported molar HOM yield estimates from isoprene up to now are $0.03 \%$ (OH oxidation) and $0.01 \%$ (ozonolysis) [21].

The newest group of compounds to be shown to produce HOM are aromatics. Following $\mathrm{OH}$ reactions with alkyl benzenes, HOM formation pathways were analyzed both experimentally as well as by quantum chemical calculations, showing that longer alkyl chains greatly enhanced the progression of autoxidation [47].

\section{Current mechanistic understanding of HOM formation}

The process of HOM formation, as defined here, requires sequential autoxidation to take place, which competes with other reaction pathways. Figure 2 provides a schematic overview of the different reactions of importance in autoxidation and $\mathrm{HOM}$ formation. The process starts by the initial formation of $\mathrm{RO}_{2}$, typically from VOC oxidation or photolysis. The fate of the peroxy radicals is a competition between uniand bimolecular reactions.

The main bimolecular reaction partners in the atmosphere are $\mathrm{NO}, \mathrm{HO}_{2}$ and other $\mathrm{RO}_{2}$. As the rate constants of all these reactions depend on the specific $\mathrm{RO}_{2}$, and since the concentrations of the reaction partners vary greatly as a function of time and location, the lifetime of $\mathrm{RO}_{2}$ with respect to this pathway is highly variable. As a rough estimate, the rate constant with $\mathrm{NO}$ is on the order of $10^{-11} \mathrm{~cm}^{3}$ molecules $^{-1}$ $\mathrm{s}^{-1}$ [48] while ambient NO concentrations range from $\sim 10^{11}$ molecules $\mathrm{cm}^{-3}$ in extremely polluted megacities to $\sim 10^{9}$ molecules $\mathrm{cm}^{-3}$ in continental background locations under sunlit conditions to $<10^{7}$ molecules $\mathrm{cm}^{-3}$ in remote locations as well as during night-time. This spans $\mathrm{RO}_{2}$ lifetimes of seconds to hours. Similar estimates can be made for the other two reaction partners. Since the rate coefficients of the reaction of $\mathrm{RO}_{2}$ with $\mathrm{HO}_{2}$ and other $\mathrm{RO}_{2}$ are believed to be around or below $10^{-11} \mathrm{~cm}^{3}$ molecules ${ }^{-1} \mathrm{~s}^{-1}$, the average lifetime of $\mathrm{RO}_{2}$ will only in rare cases be $<1 \mathrm{~s}$ in the atmosphere. Consequently, in regions where the majority of atmospheric $\mathrm{VOC}$ oxidation takes place, the $\mathrm{RO}_{2}$ lifetimes are on the order of seconds to several minutes. 


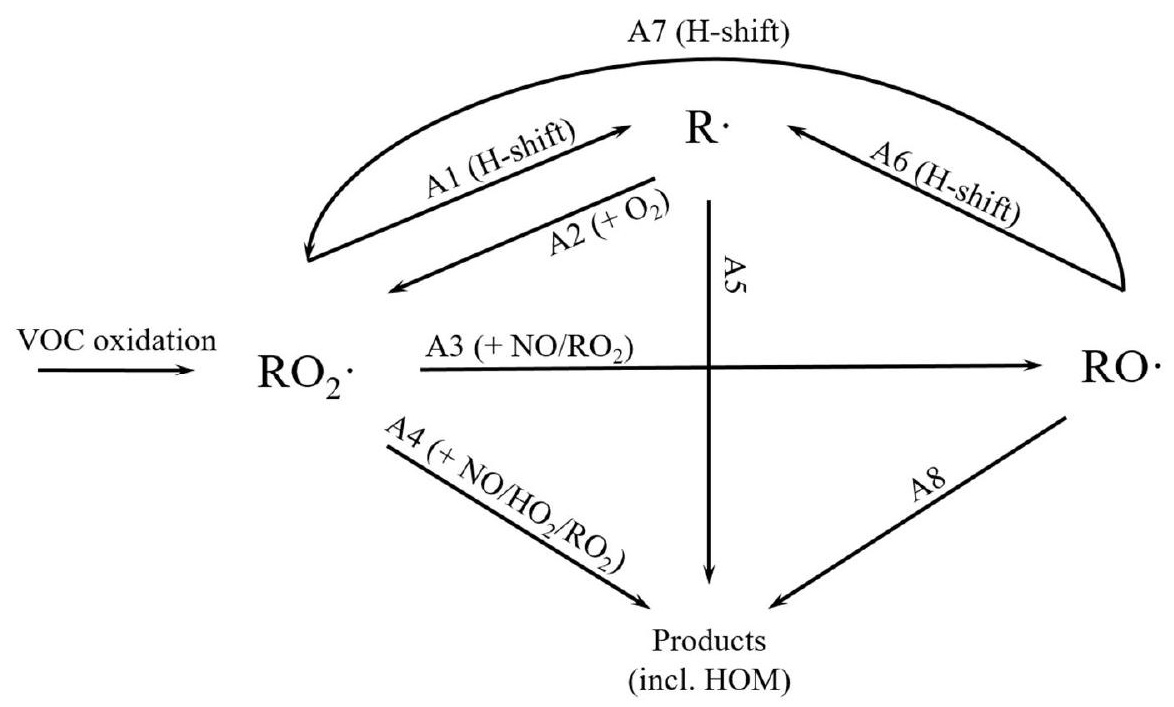

Figure 2. Schematic overview of typical radical reaction pathways forming HOM, starting from an initial $\mathrm{RO}_{2}$ from VOC oxidation. See text for details.

For unimolecular reactions, in this case $\mathrm{H}$-shift rearrangement (arrow A1 in Fig. 2), to be relevant in the atmosphere, they need to be competitive with the bimolecular reactions (A3 and A4) described above. The rate at which these $\mathrm{H}$-shifts take place is defined by the strength of the $\mathrm{C}-\mathrm{H}$ bond at the potential $\mathrm{H}$ abstraction site, as well as the steric restraint for the $\mathrm{RO}_{2}$ to reach the site. Moreover, the stability of the resulting carbon centered radical has to be considered. In most cases, the optimal configuration for the transition states of $\mathrm{H}$-shifts is by a 1,6 or 1,5 configuration. Steric restrictions can further slow down the rate of $\mathrm{H}$-shifts [41]. The strength of the $\mathrm{C}-\mathrm{H}$ bonds, for example as calculated from structure activity relationships, depend on the branching and the structure of the carbon backbone [49] and on the types of adjacent functional groups [50].

Following a successful $\mathrm{H}$-shift, a carbon-centered radical is formed. This radical will have a very short lifetime as the addition of $\mathrm{O}_{2}$ takes place with a rate of $2.5 \cdot 10^{7} \mathrm{~s}^{-1}$ [25], forming (A2 in Fig. 2) a new peroxy radical, now with two added oxygen molecules. The combination of $A 1$ and $A 2$ is what constitutes autoxidation, and these steps can proceed multiple times before the reaction chain is terminated via some other channels. In certain cases, unimolecular termination reactions (A5) can outcompete the $\mathrm{O}_{2}$ addition. For example, if the formed radical is centered on a carbon with a hydroperoxide group attached, the formation of a carbonyl and consecutive loss of $\mathrm{OH}$ will be nearly instantaneous [25]. It is also worth noting that all $\mathrm{H}$-shift reactions (A1, $A 6, A 7)$ are to some extent reversible, although depicted as one-directional in Fig. 2.

The radical chain can also be terminated via bimolecular reaction pathways, which in most cases is the primary pathway. This can occur directly via A4, but also via the alkoxy pathway A3+A8. Alkoxy radicals are expected to be very short-lived, and can either form closed shell products (A8) or undergo H-shifts $(A 6, A 7)$ to eventually form new $\mathrm{RO}_{2}$ with one 0 -atom more than the preceding $\mathrm{RO}_{2}$. This can take place either by $\mathrm{H}$-abstraction from a $\mathrm{C}-\mathrm{H}$ bond $(\mathrm{A} 6)$ followed by addition of $\mathrm{O}_{2}(\mathrm{~A} 2)$, or by directly abstracting an $\mathrm{H}$-atom from a hydroperoxide group (A7). In both cases the result is an alcohol moiety and a peroxy group. Ultimately, the termination reactions $(A 4, A 5, A 8)$ will form closed shell products that can be classified as 
HOM if they have undergone several steps of autoxidation. Depending on the pathways taken, the resulting HOM species can have similar composition to the precursor VOC with a high degree of oxygenation, but can also be organic nitrate $\left(\mathrm{RONO}_{2}\right)$ or "dimer" species (ROOR) with roughly twice the number of $\mathrm{C}$-atoms of the precursor.

\section{The chemical kinetics challenge}

The current knowledge on HOM formation by autoxidation, as outlined in the section above, is based on combined findings from the atmosphere, flow tube studies as well as chambers. However, several limitations exist, which seriously hamper the continued development of this topic, especially in the case of a truly mechanistic understanding which would allow us to make quantitative estimates of the importance of the different pathways, as outlined in Fig. 2, under various conditions.

Below we define six principal challenges for the chemical kinetics community, each of which would greatly facilitate future research within atmospheric HOM formation, but for which suitable methods do not currently exist within the HOM community. Our aim is to postulate these challenges in enough detail to spur innovative solutions from chemical kineticists, and will refrain from speculating on any possible directions through which these challenges could be tackled.

Challenge 1: The role of NO in HOM formation

In the atmosphere, NO is an important trace gas controlling the fate of peroxy radicals. At high concentrations, NO may out-compete autoxidation reactions, and even at lower concentrations it can change the $\mathrm{HOM}$ product distribution by decreasing dimers (from $\mathrm{RO}_{2}+\mathrm{RO}_{2}$ reactions) and increasing organonitrates [14]. The pathways leading to alkoxy radicals are expected to become more important with elevated NO, but how this impacts the final HOM production remains largely unknown. In other words, this is a considerable challenge which includes determining several different branching ratios, all of which will be NO concentration-dependent as well as precursor molecule-dependent.

Challenge 2: Sequential $\mathrm{OH}$ reactions versus autoxidation

The observed low volatility and high $\mathrm{O} / \mathrm{C}$ ratio of SOA constituents in the atmosphere [3] can be reached via two main routes: sequential $\mathrm{OH}$ oxidation reactions or a single reaction with an oxidant followed by autoxidation. Before the concept of atmospheric autoxidation, evolution of atmospheric VOC, OVOC and SOA were often parameterized according to an effective "OH dose" ([OH] x exposure time) [7,51]. The current challenge is to quantify the contribution to highly oxygenated species (and subsequent SOA formation) in the atmosphere from multiple oxidant reactions versus "direct" autoxidation pathways under various time scales.

Challenge 3: Accretion product ("dimer") formation

Dimer formation has been observed in several studies, both in ambient $[11,12,14]$ and laboratory $[12,14,21-23,25,27,33]$, all in line with formation via $\mathrm{RO}_{2}$ cross reactions. These $\mathrm{HOM}$ dimers have been shown to be important in the formation of new aerosol particles [19,52]. However, the structural requirements for accretion product formation from the $\mathrm{RO}_{2}+\mathrm{RO}_{2}$ reaction remains unclear. The branching ratio of the accretion product pathway from $\mathrm{RO}_{2}+\mathrm{RO}_{2}$ is likely dependent on the degree and type of functionalization of the $\mathrm{RO}_{2}$ radicals involved, but this remains highly 
speculative and needs experimental validation through systematic studies of cross-reactions between various types of $\mathrm{RO}_{2}$. It is worth noting, that the molecular compositions of these HOM dimers are not the same as for accretion products like ester dimers or hemi-acetals which have been identified in the particulate phase $[53,54]$.

Challenge 4: The role of $\mathrm{NO}_{3}$ radicals in $\mathrm{HOM}$ formation

The nitrate radical $\left(\mathrm{NO}_{3}\right)$ is the third important oxidant, in addition to $\mathrm{OH}$ and $\mathrm{O}_{3}$. The role of the nitrate radical in SOA formation has received much attention recently [55]. Findings suggest that $\mathrm{NO}_{3}$-initiated oxidation of -pinene has the potential to form considerably more SOA than the corresponding reaction with . - pinene [56]. However, until now, no studies have focused on HOM formation starting from $\mathrm{NO}_{3}$-initiated oxidation of any VOC. Monoterpene-derived HOM from nitrate radicals have been tentatively observed in the atmosphere [28], but no estimates of yields nor attribution to specific types of monoterpenes have been possible. $\mathrm{As} \mathrm{NO}_{3}$ can add to double bonds as well as abstract aldehydic $\mathrm{H}$-atoms, its role in initiating autoxidation still requires systematic studies. This will clarify the potential importance of night-time reactions for SOA formation.

Challenge 5: HOM production and detection on sub-second time scales.

While not unrelated to the other challenges, this specific challenge is an essential one, as autoxidation has been shown to take place under extremely short time scales (seconds or less). For example, during cyclohexene ozonolysis, already after $1.5 \mathrm{~s}$ the three first autoxidation steps have taken place (Fig. 3 ) and at longer time scales the product concentrations merely increase at equal ratios. Thus, for a mechanistic understanding of autoxidation, and to identify the time scales at which competing reactions can occur, faster measurement methods are required. This includes both the production and detection techniques. One specific target is to be able to quantify both forward and backward $\mathrm{H}$-shift reaction rates. 
Figure 3. Peroxy radical product distribution as a function of reaction time during cyclohexene ozonolysis in a flow tube. The relative distribution of $\mathrm{RO}_{2}$ is achieved already at the shortest measurable residence time (1.5 s). $\left[\mathrm{O}_{3}\right]=6.1 \cdot 10^{11}$ and $\left[\mathrm{C}_{6} \mathrm{H}_{10}\right]=1.25 \cdot 10^{12}$ molecules $\mathrm{cm}^{-3}$. Figure adapted from Bemdt et al. [33].

Challenge 6: Temperature dependence of HOM formation.

The atmospheric oxidation of VOC takes place over a wide range of temperatures, and H-shift reaction rates, both forward and backward, are expected to be faster at higher temperatures, possibly affecting HOM yields. In fact, all unimolecular reactions in Fig. 2, including termination reactions, may change significantly, and at significantly different rates, over the range of atmospherically relevant temperatures. As temperatures vary with location, height and time-ofday, an accurate assessment of the temperature dependence on different steps of HOM formation, as well as on the final product distribution, is required. Experiments reaching outside the range of atmospheric temperatures can also give useful mechanistic insights relevant for autoxidation in the atmosphere. However, such measurements require keeping well-defined temperature conditions nearly all the way until the products are detected, and changing temperatures without impacting the detection sensitivity of the instrument. For this purpose, the Cl-APi-TOF is not an optimal instrument.

\section{Conclusions}

Autoxidation has been shown to produce highly oxygenated molecules (HOM) under atmospheric conditions [14], and these HOM can greatly influence aerosol loadings and subsequently air quality and Earth's climate. Despite recent progress, a fundamental mechanistic understanding of HOM formation under different conditions remains elusive, to a large extent due to limitations in the instrumentation able to detect HOM and their precursors radical intermediates: currently these are still restricted to time scales of a second or more, sensitivities are hard to quantify, and they are only able to provide elemental compositions without structural information.

We have defined six specific challenges for the chemical kinetics community related to autoxidation and HOM formation. A solution to any of these challenges would facilitate a large leap forward in our ability to understand the role of HOM and SOA in the atmosphere. Consequently, understanding climate change, on time-scales of years or even hundreds of years, is to a large extent still limited by our lack of knowledge on chemical reactions occurring in millisecond time-scales.

\section{Bibliography}

1. Guenther, A. B.; Jiang, X.; Heald, C. L.; Sakulyanontvittaya, T.; Duhl, T.; Emmons, L. K.; Wang, X. Geosci. Model Dev. 2012, 5, 1471-1492.

2. $\quad$ Lamarque, J. F.; Bond, T. C.; Eyring, V.; Granier, C.; Heil, A.; Klimont, Z; Lee, D.; Liousse, C.; M ieville, A.; Owen, B.; Schultz, M. G.; Shindell, D.; Smith, S. J.; Stehfest, E.; Van Aardenne, J.; Cooper, 0. 



